\title{
RASTREAMENTO SOROLÓGICO PARA HEPATITE B EM PROFISSIONAIS DE SAÚDE NA CIDADE DE GOIÂNIA - GOIÁS
}

\author{
Marli S. Pereira de Azevedo, Divina das Dores P. Cardoso, Regina Maria \\ Bringel Martins, Roberto Ruhman Daher, Sandra Cristina T. Camarota e \\ Aristides J. Barbosa
}

\begin{abstract}
Pesquisou-se marcadores para o vírus da hepatite $B$ em profissionais de saúde, ligados a três instituições na cidade de Goiânia-Goiás. A prevalência da infecção viral encontrada foi de 23,4\% em relação aos diferentes marcadores (AgHBs, anti-HBs e anti-HBc). Entre os individuos positivos, $2,3 \%$ eram portadores do vírus e $21,1 \%$ demonstraram infecção prévia. Destes, $9,8 \%$ apresentaram como marcador, apenas o anti-HBs e 32,6\% o anti-HBc. Dezenove indivíduos relataram vacinação ao vírus, sendo que 8 soroconverteram para o anti-HBs e 6 apresentaram também o anti-HBc, e os demais não apresentaram qualquer marcador viral. A maior prevalência foi a partir dos 30 anos de idade $(p<0,05)$. O sexo masculino mostrou percentual superior ao feminino, $29,5 \%$ e $21,1 \%$ respectivamente $(p<0,05)$. Quando considerada a prevalência viral em relação a contatos com sangue elou pacientes, o percentual de positividade para contactantes foi $25,9 \%(p<0,05)$, e a maior prevalência do virus ocorreu a partir de 10 anos de trabalho $(p<0,05)$. A hemodiálise mostrou-se como setor de maior risco cujo percentual foi de $77,0 \%(p<0,05)$.
\end{abstract}

Palavras-chaves. Profissionais de saúde. Hepatite B. Fatores de risco.

$O$ virus da hepatite $B$ (VHB) ocorre em todos os continentes com prevalência variável e, mundialmente, estima-se que existam 300 milhões de pessoas portadoras do agente 524 .

Este vírus pode causar num organismo tanto infecção inaparente quanto sintomática e pode evoluir da condição de infecção aguda para crônica, persistente ou ativa, e em piores condiçôes, para cirrose e/ou câncer primário de fígado ${ }^{1524} 29$. Todos estes aspectos levam a uma perda da produtividade e aumento de gastos públicos.

A disseminação do agente na natureza ocorre por via vertical ${ }^{724}$, sexual ${ }^{171827}$ e parenteral $^{5}$, e em alguns países o índice atinge cerca de $60,0 \%$, com a infecção provavelmente adquirida por via perinatal $^{22}$.

A transmissão do VHB por via parenteral está bem estabelecida, e condiciona a existência de

\footnotetext{
Laboratório de Virologia do Instituto de Patologia Tropical e Saúde Pública, Universidade Federal de Goiás, Goiânia, GO. Suporte Financeiro: Conselho de Ciência e Tecnologia do Estado de Goiás - CONCITEG.

Endereço para correspondência: Profa Marli S. P. Azevedo. Faculdade de Farmácia/UFG. Pça. Universitária s/n, Setor Universitário. Caixa Postal 315, 74605-020 Goiânia, GO. Recebido para publicação em $13 / 10 / 93$.
}

alguns grupos de risco pelo contato com sangue, produtos sanguíneos ou outros fluidos corporais, seja pela ocupação, doença ou hábitos pessoais ${ }^{11}$. Os casos de hepatite nesta população são na maioria brandos ou subclínicos ${ }^{10}$, o que os torna disseminadores silenciosos do vírus na natureza e, desta forma, medidas de prevenção são necessárias a fim de interromper o elo de transmissão.

Estudos realizados em nossa região mostram percentuais variáveis de prevalência para o vírus em diferentes tipos populacionais ${ }^{3}{ }^{16}$.

Em estudo conduzido em profissionais de saúde do Rio de Janeiro, Coelho e cols encontraram índices de $40 \%$ para cirurgiões e $36,4 \%$ para profissionais de hemodiálise, e concluíram que a vacinação é indicada para este segmento populacional $^{9}$.

Neste trabalho, analisamos a prevalência do VHB em um segmento populacional ligado à saúde, o que nos permitiu avaliar e evidenciar fatores $\mathrm{e}$ setores de maior risco para esta população. Pretendemos também, fornecer informações à saúde pública, que somadas, poderão servir de subsídios a futuras, necessárias e eficazes medidas de prevenção, entre elas a vacinação. 
Azevedo MSP, Cardoso DDP, Martins RMB, Daher RR, Camarota SCT, Barbosa AJ. Rastreamento sorológico para hepatite $B$ em profissionais de saúde na cidade de Goiânia - Goiás. Revista da Sociedade Brasileira de Medicina Tropical 27:157-162, jul-set, 1994.

\section{MATERIAL E MÉTODOS}

Entre maio/1989 e abril/1990, foram coletadas 625 amostras sanguíneas em trabalhadores de três instituições de saúde na cidade de Goiânia, GO, com a finalidade de detectar marcadores para o vírus de Hepatite B (VHB).

Não houve seleção de grupos nesta população que pudesse representar maior grau de exposição ao vírus, sendo aceito todo e qualquer indivíduo com participação voluntária.

De cada pessoa, foram coletadas e anotadas informações, em formulário pertinente, desde dados pessoais a fatores de risco, como história anterior e familiar de hepatite, multiplicidade de parceiros, transfusão sanguínea, uso de acupuntura, tatuagem, drogas injetáveis, doenças sexualmente transmissíveis, além de vacinação para o VHB.

Todas as amostras foram analisadas, visando deteç̧ão do AgHBs, anti-HBs e anti-HBc. Parte das amostras negativas (10\%) e todas as positivas foram enviadas ao Centro Nacional para Hepatites Virais (CNHV) - Fundação Oswaldo Cruz, coma finalidade de corroboração dos resultados. As amostras AgHBs $\mathrm{e}$ anti-HBc positivas foram, ainda, testadas para $\mathrm{o}$ anti-HBc-IgM, naquela instituição.

A metodologia utilizada para análise dos marcadores virais foi o ensaio imunoenzimático (ELISA), conforme preconizado por Voller e cols, $1978^{25}$ e modificado de acordo com Camargo e cols, $1987^{2}$.

Para a análise do AgHBs, anti-HBs, anti-HBc$\mathrm{IgM}$ foram utilizados kits preparados ecedidos pelo Laboratório do CNHV do Departamento de Virologia da Fundação Oswaldo Cruz. Quanto ao anti-HBc, foi utilizado kit comercial da Organon Teknica-Hepanostika anti-HBc Microelisa System. A leitura das reações foi realizada visualmente e em leitor Microwell System-Reader 210 (Organon Teknika) com filtros de 450 ou $492 \mathrm{~nm}$.

A análise estatística foi realizada aplicando-se o teste do Qui-quadrado $\left(\mathrm{X}^{2}\right)$ com intervalo de confiança de $95,0 \%$.

\section{RESULTADOS}

Dos 625 profissionais de saúde participantes do estudo, $146(23,4 \%)$ apresentaram marcadores para - VHE (rabela 1). Observamos que $14(2,3 \%)$ eram portadores do vírus, sendo que $8(1,3 \%)$ apresentaram também anti-HBc IgM, e 132(21,1\%) mostraram apenas anticorpos ao vírus (anti-HBs/ anti-HBc). Desta população (625 indivíduos) 19 relataram vacinação para o VHB prévia e, destes, 8 apresentaram anti-HBs apenas e 6 também antiHBc-total.

Tabela 1 - Condição sorológica de 625* profissionais de saúde de Goiânia, GO, considerando os diferentes marcadores pesquisados.

\begin{tabular}{lcc}
\hline Condições/marcadores & $\begin{array}{c}\mathrm{N}^{0} \text { positivos/ } \\
\mathrm{n}^{0} \text { examinados }\end{array}$ & $\%$ \\
\hline $\begin{array}{l}\text { Portadores do vírus } \\
\text { (AgHBs e/ou anti-HBc-IgM }\end{array}$ & $14 / 625$ & 2,3 \\
$\begin{array}{l}\text { Infecção prévia } \\
\text { (anti-HBs e/ou anti-HBc) }\end{array}$ & $132 / 625$ & 21,1 \\
\hline
\end{tabular}

Total

$146 / 625$ 23,4

* 19 destes indivíduos relataram vacinação prévia, sendo que 8 soroconverteram para anti-HBs e 6 apresentaram também anti-HBc.

A Tabela 2 apresenta a distribuição de positividade por faixa etária. Nota-se um aumento da prevalência viral a partir dos 30 anos de idade $(\mathrm{p}<0,05)$.

O sexo masculino apresentou maior percentual de positividade $(29,5 \%)$ em relação ao feminino $(21,1 \%)$ $(p<0,05)$, o que pode ser visto na Tabela 3.

Quando considerado o aspecto contato com sangue e/ou pacientes (Tabela 4), os percentuais de positividade foram significativamente maiores

Tabela 2 - Distribuição percentual da positividade ao virus da hepatite $B(V H B)$ em profissionais de saúde de Goiânia. GO, de acordo com a faixa etária.

\begin{tabular}{ccc}
\hline $\begin{array}{c}\text { Faixa etária } \\
\text { anos }\end{array}$ & $\begin{array}{c}\mathrm{N}^{0} \text { positivos/ } \\
\mathrm{n}^{0} \text { examinados }\end{array}$ & $\%$ \\
\hline $15-30$ & $35 / 208$ & 16,9 \\
$30-45$ & $75 / 295$ & 25,5 \\
$45-60$ & $35 / 116$ & 30,1 \\
$>60$ & $01 / 006$ & 16,6 \\
\hline Total & $146 / 625$ & 23,4 \\
\hline
\end{tabular}

$\mathrm{X}^{2}=8,81 ; \mathrm{p}<0,05$ 
Azevedo MSP, Cardoso DDP, Martins RMB, Daher RR, Camarota SCT, Barbosa AJ. Rastreamento sorológico para hepatite $B$ em profissionais de saúde na cidade de Goiania - Goiás. Revista da Sociedade Brasileira de Medicina Tropical 27:157-162, jul-set, 1994.

$(p<0,05)$ para os contactantes.

A Figura 1 mostra a positividade em relação ao tempo de trabalho na área de saúde. Observou-se que a maior prevalência do vírus ocorreu a partir de 10 anos de tempo de trabalho $(p<0,05)$.

A positividade em relação ao local de trabalho é mostrada na Figura 2. A hemodiálise, o setor de maior percentual $(77,0 \%)$, foi estatisticamente significante $(p<0,05)$.

Tabela 3 - Prevalência parao vírus da hepatite $B(V H B)$ em profissionais de salide de Goiânia, GO, considerando sexo.

\begin{tabular}{lcc}
\hline Sexo & $\begin{array}{c}\mathrm{N}^{0} \text { positivos/ } \\
\mathrm{n}^{0} \text { examinados }\end{array}$ & $\%$ \\
\hline Feminino & $97 / 459$ & 21,1 \\
Masculino & $49 / 166$ & 29,5 \\
\hline Total & $146 / 625$ & 23,4 \\
\hline
\end{tabular}

$X^{2}=4,83 ; p<0,05$

Tabela 4 - Presença de marcadores para o vírus da hepatite $B(\mathrm{VHB})$ em profissionais de saude de Goiania, GO, considerando os fatores de risco relacionados a contatos com sangue ef ou pacientes.

\begin{tabular}{lcc}
\hline Profissionais & $\begin{array}{c}\mathrm{N}^{\circ} \text { positivos/ } \\
\mathrm{n}^{0} \text { examinados }\end{array}$ & $\%$ \\
\hline Contactantes & $109 / 420$ & 25,9 \\
Não contactantes & $37 / 205$ & 18,0 \\
\hline Total & $146 / 625$ & 23,4 \\
\hline
\end{tabular}

$X^{2}=4,79 ; p<0,05$

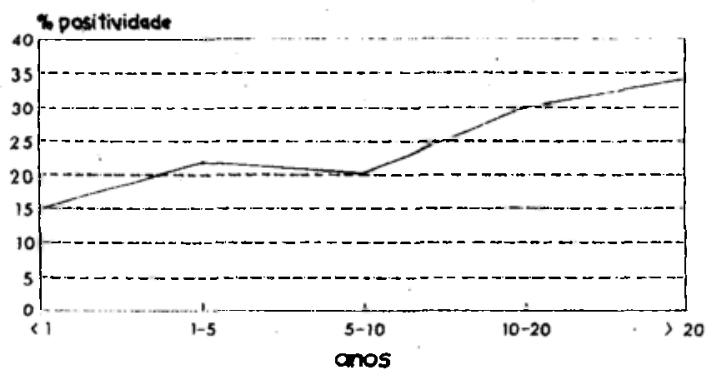

$\ldots \times 2-11,03 \mathrm{p}<0,05$

Figura 1 - Positividade para VHB por anos de trabalho ligado à saúde.

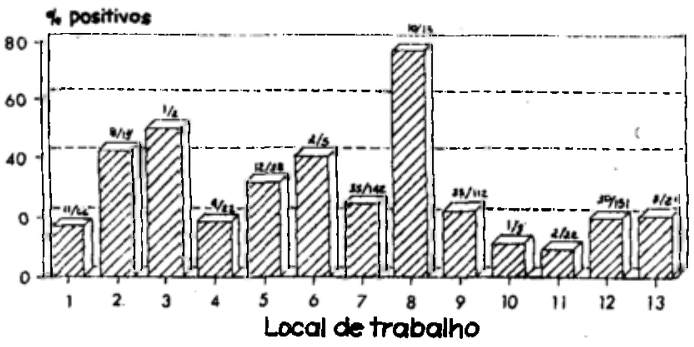

$P Z 7 \times 2=31,8 \quad p<0,05$

1. administração

2. ambulatório

3. banco de sangue

4. centro de material

5. clínica cirúrgica
6. emergência 10. raio-X

7. enfermarias 11. sala de aula

8. hemodiálise 12. serviços gerais

9. laboratório 13. UTI

Figura 2 - Positividade para VHB em profissionais de saúde considerando local de trabalho.

\section{DISCUSSÃO}

A prevalência do vírus da hepatite B (VHB), pelos marcadores (AgHBs, anti-HBs e anti-HBc) em nosso estudo, foi de $23,4 \%$, contrastando com os de outras populações por nós estudadas, como $12,9 \%$ para primodoadores de sangue ${ }^{16}$ e $6,1 \%$ para população feminina urbana ${ }^{3}$.

Entre os indivíduos positivos, $2,3 \%$ eram portadores do vírus, demonstrável pela presença de anti-HBc IgM, isoladamente ou em associação com AgHBs, e $21 \%$ mostraram uma condição de infecção prévia pela presença de anti-HBc e/ou anti-HBs (Tabela 1). Esta condição, embora considerada satisfatória em termos de seguimento clínico/ laboratorial, à luz dos conhecimentos atuais, é discutível, pois tem-se visto que indivíduos antiHBs/anti-HBc positivos, ou mesmo na ausência de qualquer marcador, quando analisados por técnicas metodológicas moleculares, podem mostrar o DNA viral $^{48}$. Assim sendo, para que esta prevalência não seja subestimada, poder-se-ia usar técnicas mais sensíveis como Hibridização e Reação em Cadeia de Polimerase (PCR). No entanto, por suas dificuldades e alto custo, estas técnicas dificilmente podem vir a fazer parte da rotina de rastreamento ${ }^{21}$. Destes indivíduos (132), 13 apresentaram como marcador apenas anti-HBs, e 43 anti-HBc. Existe o consenso de que a presença isolada do anti-HBs indica exposição a baixas doses do $\mathrm{AgHBs}^{11}$ ou, ainda, 
Azevedo MSP, Cardoso DDP, Martins RMB, Daher RR, Camarota SCT, Barbosa AJ. Rastreamento sorológico para hepatite $B$ em profissionais de saúde na cidade de Goiânia - Goiás. Revista da Sociedade Brasileira de Medicina Tropical 27:157-162, jul-set, 1994.

uma situação de falsos positivos ${ }^{28}$. Já a ocorrência apenas do anti-HBc é explicada pelo fato de que este, talvez permaneça em níveis detectáveis por mais tempo e, assim, vem sendo considerado como melhor marcador da intensidade de exposição ao sangue $^{11}$, embora não exclua a necessidade de vacinação, pois a imunidade protetora parece ser conferida pelo anti-HBs ${ }^{28}$.

Houve 19 indivíduos que relataram vacinação ao vírus, sendo que 8 soroconverteram para antiHBs e 6 apresentaram também anti-HBc e os demais não apresentaram quaisquer marcadores, o que pode refletir uma infecção prévia, obviamente não triada, ou ainda uma falha vacinal.

A análise em relação à faixa etária mostra um percentual de positividade mais elevado a partir de 30 anos. Este fato pode ser correlacionado a uma maior atividade sexual, além da condição de definição profissional, o que tem como censeqüència, um maior tempo de contato ao ambiente de risco, o que pode ser comprovado pelo fato desta população mostrar maior prevalência de infecção a partir de 10 anos de atuação (Figura 1).

O sexo masculino mostrcu prevalência significativamente maior em relação ao feminino (Tabela 3), o que pode ser devido, pelo menos em parte, a uma maior mobilidade sexual masculina. Além disso, alguns autores aventam uma maior predisposição do sexo masculino ${ }^{13}$ ao VHB e até mesmo à cronicidade da doença, já que a expressão viral de alguns genes parece estar sob controle de hormônios esteróides ${ }^{24}$.

A prevalência viral em relação a contatos com sangue e/ou pacientes (Tabela 4) evidencia uma maior probabilidade de infecção pelo vírus. Quando analisamos a circulação viral em relação a local de trabalho, observamos que o setor de hemodiálise foi o de maior prevalência (Figura 2), muito embora o pessoal ligado ao setor de limpeza e outros serviços congêneres, também, mostrasse índices importantes de positividade. Acreditamos que, entre outros fatores, uma possível explanação seja, além do risco inerente ao sangue, a falta de conhecimentos básicos em relação ao risco da infecção pelo vírus, principalmente entre o pessoal ligado ao serviço geral daquelas instituições.
Admite-se que precauções a fim de prevenir ferimentos, uso de barreiras protetoras e a conscientização do potencial infeccioso do sangue e fluidos corporais sejam estratégias que, aliadas à vacinação, podem alterar a atual condição dos profissionais de saúde ${ }^{5}$, uma vez que a hepatite $B$ constitui doença de difícil controle, justificado pela existência de grupos que não são atingidos por programas de imunização, como homossexuais, viciados em drogas e pessoas expostas a contatos heterossexuais ${ }^{6}{ }^{19}$.

A detecção dos portadores do VHB tem implicações diagnósticas, terapêuticas e epidemiológicas ${ }^{28}$, além do risco, não bem definido, que o profissional de saúde portador representa para seus pacientes 12027 .

Em vista dos resultados observados, torna-se evidente que medidas de proteção para esta população fazem-se necessárias e a vacinação sistemática, com triagem prévia dos marcadores no serviço de saúde, deve ser recomendada ${ }^{12142328}$.

\section{SUMMARY}

Markers for hepatitis $B$ virus in health care workers were analysed in 625 employees of three institutions in Goiania city. The virus prevalence was $23.4 \%$ related to different markers (AgHBs, anit-HBs and anti-HBc). Among the positive individuals, $1.3 \%$ presented acute infection, $1.0 \%$ were virus carriers and $21.1 \%$ presented only anti-HBs and $32.6 \%$ only anti-HBc as markers. There were 19 reports of virus vaccination. Eight of them seroconverted for anti-HBs and 6 were positive for both anti-HBc and anti-HBs. Higher prevalence rates were found above 30 year of age $(p<0.05)$. The male and female prevalence rates were $29.5 \%$ and $21.1 \%(p<0.05)$ respectively. The virus prevalence rates among blood and patient contacts were $25.9 \%(p<0.05)$ and the highest prevalence was found in persons followed up for at least ten years $(p<0.05)$. The greatest risk section has shown to be hemodialysis unit personnel with a percentual of $77 \%(p<0.05)$.

Key-words: Health care workers. Hepatitis B. Risk factors. 
Azevedo MSP, Cardoso DDP, Martins RMB, Daher RR, Camarota SCT, Barbosa AJ. Rastreamento sorológico para hepatite $B$ em profissionais de saüde na cidade de Goiânia - Goiás. Revista da Sociedade Brasileira de Medicina Tropical 27:157-162, jul-set, 1994.

\section{REFERÊNCIAS BIBLIOGRÁFICAS}

1. Alter HJ, Chalmers TC, Freeman BM, Lunceford JL, Lewis TL, Holland PV, Pizzo PA, Meyer III WJ. Health care workers positive for Hepatitis B surface antigen, are their contacts at risk? New England Journal of Medicine 292:454-457, 1975.

2. Camargo IF, Gaspar AMC, Yoshida CFT. Comparative ELISA reagents for detection of Hepatitis B surface antigen ( $\mathrm{HBsAg}$ ). Memórias do Instituto Oswaldo Cruz 82:181-187, 1987.

3. Cardoso DDP, Azevedo MSP, Martins RMB, Barbosa AJ, Camarota SCT. Soroprevalência para infecção pelo vírus da Hepatite $B$ pelos marcadores AgHBs e anti-HBs em população feminina de área urbana de Goiânia-GO. Revista de Patologia Tropical 19:135-141, 1990.

4. Carloni $G$, Defini $C$, Colloca S, Alfani E, Taliane G, De Bac C. Incidence of Hepatitis B virus DNAPolymerase in sera of Italian asymptomatic carriers with serological markers of HBV. Archives of Virology 87:1-2, 1986.

5. Centers For Disease Control. Update: Universal Precaution for prevention of transmission of Human Immunodeficiency Virus, Hepatitis B, and other bloodborne pathogens in health-care setting. Leds from the MMWR vol37 n 24 1988. Journal American Medical Association 260:462-465, 1988.

6. Centers For Disease Control. Changing patterns of groups at high risk for Hepatitis B in the United States. Leds from the MMWR vol 37 n 26, 281988. Journal American Medical Association 260:761770, 1988.

7. Centers For Disease Control. Prevention of perinatal transmission of Hepatitis B virus. Prenatal screening of all pregnat women for Hepatitis B surface antigen. Morbidity and Mortality Weekly Report 37:341351, 1988.

8. Chemin I, Banginski I, Petit MA, Zoulim F, Pichoud C, Capel F, Hantz O, Trepo C. Correlation between HBV DNA detection by polymerase Chain Reaction and Pre-S1 antigenemia in symptomatic and asymptomatic Hepatitis B virus infections. Journal of Medicine Virology 33:51-57, 1991.

9. Coelho HSM, Artemenko SRT, Martin CN, Carvalho DM, Valente J, Rodrigues EC, Alves LS, Martins MLM. Prevalência da infecção pelo vírus B na comunidade hospitalar. Revista da Sociedade Brasileira de Medicina Tropical 23:71-76, 1990.

10. Denes AE, Smith JT, Maynard JE. Hepatitis B infection in physicians. Results os nationwide seroepidemiologic survey. Journal American Medical Association 239:210-212, 1978.

11. Diestang JL, Ryan DM. Occupational exposure to Hepatitis B virus in hospital personnel: Infection or imunization? American Journal of Epidemiology 115:26-39, 1982.

12. Follet EAC, Symington IS, Cameron MG. Experience with Hepatitis $B$ vaccination in nurses in a hospital for mentally handicapped. Lancet II 8561:728-732, 1987.

13. Gok HT, Chan YW, Wong LYM, Kong KH, Oon CJ, Guan R. The prevalence of Hepatitis B virus markers in dental personnel in Singapore. Transactions of the Royal Society of Tropical Medicine and Hygiene 82:908-910, 1988.

14. Jonsson JB. Cost-benefit analysis of Hepatitis B vaccination. Postgraduate Medical Journal 63(supl 2):27-32, 1987.

15. Leite AMPS, Mendes TF. Vírus B e carcinoma hepatocelular. Moderna Hepatologia 1:22-23, 1987.

16. Martelli CMT, Andrade ALSS, Cardoso DDP, Sousa LCS, Silva SA, Souza MA, Zicker F. Soroprevalência e fatores de risco para a infecção pelo vírus da Hepatite B pelos marcadores AgHBs e anti-HBs em prisioneiros e primodoares de sangue. Revista de Saúde Pública de São Paulo 24:270-276, 1990.

17. Mendes TF, Cruz PRS, Pitella MM, Mexas PPF, Podkamen N, Herbet B. Transmissão sexual do vírus da Hepatite B. Moderna Hepatologia 3:1-6, 1982.

18. Papaevangelou G, Roumeliotou A, Tassopoulos $\mathrm{N}$, Kolaitis N, Stathopoulou P. Source of infection due to Hepatitis B in Greece. The Journal of Infections Diseases 147:987-989, 1983.

19. Recommendations of the Immunization Practices Advisory Commitee. Update on Hepatitis B prevention. Annals of Internal Medicine 107:353$357,1987$.

20. Sherlock S. The natural history of Hepatitis B. Postgraduate Medical Journal 63:7-11, 1987.

21. Shin LN, Sheu JC, Wang JT, Huang GT, Yang PM, Lee HS, Sung JL, Wang TH, Chen DS. Serum Hepatitis B virus DNA in health HBsAg-negative chinese adults evaluated by Polymerase Chain Reaction. Journal of Medical Virology 32:257-260, 1990.

22. Snydman DR. Hepatitis in pregnancy. New England Journal of Medicine 312:1398-1401, 1985.

23. Steketee RW, Ziarnik ME, Davis JP. Sororesponse to Hepatitis $B$ vaccine in patients and staff of renal 
Azevedo MSP, Cardoso DDP, Martins RMB, Daher RR, Camarota SCT, Barbosa AJ. Rastreamento sorológico para hepatite B em profissionais de saúde na cidade de Goiânia - Goiás. Revista da Sociedade Brasileira de Medicina Tropical 27:157-162, jul-set, 1994.

dialysis centers, Wisconsin. American Journal of Epidemiology 127:772-782, 1988.

24. Tiollais P, Buendia MA. Hepatitis B virus. Scientific American 4:48-54, 1991.

25. Voller A, Bartlett A, Bidwell DE. Enzyme immunoassays with special reference to ELISA techniques. Journal of Clinical Pathology 31:507520,1978

26. West DJ. The Hepatitis B infection among health profissionals in the United States: A review. The American Journal of Medical Sciences 287:26-33, 1984.

27. Wilkinson R. Hepatitis B as a sexually transmitted disease in a black south African population. South African Medical Journal 65:954-955, 1984.

28. Zanalda BC, Manterola AC, Lestrem MD, Frider JB, Zocchi GA, Fainboim H, Clua GI, Amor E. Prevalencia del anticuerpo contra el virus de la Hepatitis B (anti-HBc) en personal hospitalario de Buenos Aires. Boletin de la Oficina Sanitaria Panamericana 108:16-25, 1990.

29. Zuckerman AJ, Harrison TJ. Hepatitis B virus chronic liver disease and hepatocellular carcinoma. Postgraduate Medical Journal 63(supl 2):13-19, 1987. 